\title{
Solution Structure of an RNA Internal Loop with Three Consecutive Sheared GA Pairs
}

Gang Chen, Brent M. Znosko, Scott D. Kennedy, Thomas R. Krugh, Douglas H. Turner

Supporting Information 
Table S1. List of Distance and Dihedral Angle Restraints Used for Modeling.

\begin{tabular}{llll}
\hline & \multicolumn{2}{c}{ Hydrogen bond restraits } \\
Atom 1 & Atom 2 & Lower limit & Upper limit \\
1:G5'_1:H1 & $1: \mathrm{C} \_19: \mathrm{N} 3$ & 1.800 & 2.500 \\
1:G5'_1:O6 & 1:C_19:H42 & 1.800 & 2.500 \\
1:G5'_1:H22 & 1:C_19:O2 & 1.800 & 2.500 \\
1:G_2:H1 & 1:C_18:N3 & 1.800 & 2.500 \\
1:G_2:O6 & 1:C_18:H42 & 1.800 & 2.500 \\
1:G_2:H22 & 1:C_18:O2 & 1.800 & 2.500 \\
1:G_7:H1 & 1:C_13:N3 & 1.800 & 2.500 \\
1:G_7:O6 & 1:C_13:H42 & 1.800 & 2.500 \\
1:G_7:H22 & 1:C_13:O2 & 1.800 & 2.500 \\
1:G_8:H1 & 1:C_12:N3 & 1.800 & 2.500 \\
1:G_8:O6 & 1:C_12:H42 & 1.800 & 2.500 \\
1:G_8:H22 & 1:C_12:O2 & 1.800 & 2.500 \\
1:C_9:N3 & 1:G5'_11:H1 & 1.800 & 2.500 \\
1:C_9:H42 & 1:G5'_11:O6 & 1.800 & 2.500 \\
1:C_9:O2 & 1:G5'_11:H22 & 1.800 & 2.500
\end{tabular}

Distance restraints, upper limits loosened with the note *

\begin{tabular}{|c|c|c|c|c|c|}
\hline \multirow{2}{*}{ Hydrogen 1} & Hydrogen 2 & lower limit & upper limit & NMR distance & note \\
\hline & \multicolumn{5}{|c|}{ from SNOESY spectrum with $150 \mathrm{~ms}$ mixing time } \\
\hline 1:U_3:H3 & $1: G \_2: H 1$ & 1.750 & 4.082 & 2.916 & \\
\hline $1: G \_17: H 1$ & $1: \mathrm{G} \_2: \mathrm{H} 1$ & 1.940 & 4.527 & 3.234 & \\
\hline 1:U_3:H3 & $1: G \_4: H 1$ & 2.828 & 6.599 & 4.713 & \\
\hline $1: G_{-} 4: \mathrm{H} 1$ & 1:G_5:H1 & 2.340 & 5.459 & 3.899 & \\
\hline 1:G_14:H1 & 1:G_5:H1 & 2.500 & 7.000 & - & $*$ \\
\hline $1: \mathrm{U} \_3: \mathrm{H} 3$ & 1:C_18:H42 & 2.252 & 5.255 & 3.754 & * \\
\hline 1:U_3:H3 & 1:C_18:H41 & 2.166 & 7.000 & 3.609 & \\
\hline 1:P3'_20:H6 & 1:C_19:H41 & 1.942 & 4.531 & 3.237 & \\
\hline 1:P3'_20:H6 & 1:C_19:H42 & 1.780 & 4.153 & 2.966 & \\
\hline 1:G5'_11:H1 & 1:U3'_10:H1' & 2.404 & 5.610 & 4.007 & \\
\hline 1:G_7:H1 & 1:G_14:H1' & 1.864 & 4.305 & 3.107 & \\
\hline 1:G_17:H1 & $1: G_{-} 4: H 1^{\prime}$ & 1.442 & 3.364 & 2.403 & \\
\hline 1:P3'_20:H1' & 1:G5'_1:H1 & 2.125 & 4.958 & 3.542 & \\
\hline 1:G_2:H1 & 1:U_3:H1' & 2.068 & 4.825 & 3.446 & \\
\hline 1:U_3:H2' & 1:G_17:H1 & 2.410 & 5.624 & 4.017 & \\
\hline 1:G_5:H1 & 1:G_14:H2' & 2.502 & 5.838 & 4.170 & \\
\hline 1:P3'_20:H6 & 1:G5'_1:H1 & 2.217 & 5.172 & 3.695 & \\
\hline
\end{tabular}

from $\mathrm{D}_{\mathbf{2}} \mathrm{O}$ NOESY spectrum with $100 \mathrm{~ms}$ mixing time unless otherwise noted

$\begin{array}{llllll}1: G \_2: H 1^{\prime} & 1: G 5^{\prime} \_1: H 2^{\prime} & 2.798 & 5.196 & 3.997 & 200 \mathrm{~ms} \\ 1: G \_2: H 3^{\prime} & 1: U_{-} 3: H 5 & 2.188 & 4.064 & 3.126 & 200 \mathrm{~ms}\end{array}$




\begin{tabular}{|c|c|c|c|c|c|}
\hline $1: G \_5: H 8$ & $1: G \_4: H 8$ & 2.360 & 6.000 & 3.371 & $* 200 \mathrm{~ms}$ \\
\hline 1:G_17:H8 & 1:C_18:H5 & 3.294 & 6.000 & 4.706 & $200 \mathrm{~ms}$ \\
\hline $1: G \_17: H 8$ & 1:C_18:H6 & 3.268 & 6.000 & 4.668 & $200 \mathrm{~ms}$ \\
\hline 1:A_15:H2 & 1:A_16:H2 & 3.493 & 6.000 & 4.99 & $200 \mathrm{~ms}$ \\
\hline 1:A_15:H2 & 1:A_6:H8 & 3.839 & 6.000 & 5.484 & $200 \mathrm{~ms}$ \\
\hline 1:G_7:H8 & 1:A_6:H8 & 2.557 & 4.749 & 3.653 & $200 \mathrm{~ms}$ \\
\hline $1: \mathrm{G} \_7: \mathrm{H} 2^{\prime}$ & $1: G \_8: H 1^{\prime}$ & 2.614 & 4.855 & 3.735 & $200 \mathrm{~ms}$ \\
\hline 1:G_8:H2' & 1:C_9:H5 & 2.988 & 5.549 & 4.268 & $200 \mathrm{~ms}$ \\
\hline 1:G5'_11:H8 & 1:C_12:H5 & 3.481 & 6.000 & 4.973 & $200 \mathrm{~ms}$ \\
\hline 1:C_12:H1' & 1:G5'_11:H2' & 3.281 & 6.000 & 4.687 & $200 \mathrm{~ms}$ \\
\hline 1:A_16:H1' & 1:A_15:H2' & 3.057 & 5.677 & 4.367 & $200 \mathrm{~ms}$ \\
\hline 1:G5'_1:H8 & 1:G5'_1:H1' & 2.819 & 5.235 & 4.027 & \\
\hline 1:G5'_1:H8 & 1:G5'_1:H2' & 2.680 & 4.977 & 3.829 & \\
\hline 1:G5'_1:H8 & 1:G5'_1:H3' & 2.309 & 4.288 & 3.298 & \\
\hline 1:G5'_1:H1' & 1:G5'_1:H3' & 2.703 & 5.020 & 3.862 & \\
\hline 1:G5'_1:H1' & 1:G5'_1:H4' & 2.318 & 4.305 & 3.312 & \\
\hline 1:G5'_1:H2' & 1:G5'_1:H1' & 1.922 & 3.570 & 2.746 & \\
\hline 1:G5'_1:H3' & $1: G \_2: H 8$ & 2.040 & 3.788 & 2.914 & \\
\hline 1:G_2:H8 & 1:G5'_1:H1' & 2.966 & 5.508 & 4.237 & $200 \mathrm{~ms}$ \\
\hline 1:G_2:H8 & 1:G5'_1:H2' & 1.683 & 3.125 & 2.404 & \\
\hline 1:G_2:H8 & 1:G_2:H1' & 2.978 & 5.530 & 4.254 & \\
\hline 1:G_2:H8 & 1:G_2:H2' & 2.831 & 5.257 & 4.044 & $200 \mathrm{~ms}$ \\
\hline 1:G_2:H1' & 1:G_2:H2' & 1.975 & 3.668 & 2.821 & \\
\hline 1:G_2:H1' & 1:G_2:H3' & 2.798 & 5.196 & 3.997 & \\
\hline 1:G_2:H1' & $1: G \_2: H 4 '$ & 2.320 & 4.309 & 3.314 & \\
\hline 1:G_2:H3' & 1:G_2:H8 & 2.247 & 4.172 & 3.209 & \\
\hline 1:G_2:H3' & 1:U_3:H6 & 1.991 & 3.697 & 2.844 & \\
\hline 1:U_3:H5 & 1:G_2:H8 & 2.493 & 4.629 & 3.561 & \\
\hline 1:U_3:H6 & 1:G_2:H1' & 3.087 & 5.733 & 4.410 & $200 \mathrm{~ms}$ \\
\hline 1:U_3:H6 & 1:G_2:H2' & 1.745 & 3.241 & 2.493 & \\
\hline 1:U_3:H6 & $1: \mathrm{U} \_3: \mathrm{H} 2^{\prime}$ & 2.728 & 5.066 & 3.897 & $200 \mathrm{~ms}$ \\
\hline 1:U_3:H6 & 1:U_3:H5 & 1.826 & 3.391 & 2.608 & \\
\hline $1: \mathrm{U} \_3: \mathrm{H} 1^{\prime}$ & $1: G \_2: H 2 '$ & 2.431 & 4.516 & 3.474 & \\
\hline 1:U_3:H1' & 1:U_3:H6 & 2.365 & 4.392 & 3.378 & \\
\hline 1:U_3:H1' & 1:U_3:H3' & 2.350 & 4.363 & 3.357 & \\
\hline 1:U_3:H1' & $1: G \_4: H 8$ & 2.563 & 6.000 & 3.661 & $*$ \\
\hline $1: \mathrm{U} \_3: \mathrm{H} 2^{\prime}$ & 1:U_3:H1' & 1.919 & 3.563 & 2.741 & \\
\hline 1:U_3:H3' & 1:U_3:H6 & 2.210 & 4.104 & 3.157 & \\
\hline 1:G_4:H8 & 1:U_3:H2' & 1.717 & 3.188 & 2.453 & \\
\hline $1: G \_4: H 8$ & 1:U_3:H3' & 1.958 & 3.636 & 2.797 & \\
\hline $1: G \_4: H 8$ & $1: G \_4: H 2 '$ & 2.844 & 5.282 & 4.063 & \\
\hline 1:G_4:H8 & $1: G \_4: H 3 '$ & 2.188 & 4.063 & 3.125 & \\
\hline 1:G_4:H1' & 1:U_3:H2' & 2.192 & 4.071 & 3.131 & \\
\hline $1: \mathrm{G} \_4: \mathrm{H} 1^{\prime}$ & $1: G \_4: H 8$ & 2.796 & 5.192 & 3.994 & \\
\hline $1: G_{-} 4: H 1^{\prime}$ & $1: G \_4: H 2 '$ & 1.857 & 3.449 & 2.653 & \\
\hline 1:G_4:H1' & $1: G \_4: H 4 '$ & 2.238 & 4.156 & 3.197 & \\
\hline 1:G_5:H8 & $1: G \_4: H 2 '$ & 2.730 & 5.071 & 3.900 & \\
\hline 1:G_5:H8 & 1:G_4:H3' & 2.408 & 4.473 & 3.441 & \\
\hline
\end{tabular}




\begin{tabular}{|c|c|c|c|c|c|}
\hline $1: G \_5: H 8$ & $1: G \_5: H 2 '$ & 1.432 & 2.659 & 2.045 & \\
\hline 1:G_5:H1' & $1: G \_5: H 8$ & 3.290 & 6.000 & 4.699 & \\
\hline 1:G_5:H2' & 1:G_5:H1' & 2.232 & 4.144 & 3.188 & \\
\hline 1:G_5:H3' & 1:G_5:H8 & 2.462 & 4.573 & 3.518 & \\
\hline 1:A_6:H2 & $1: G_{-} 7: H 1 '$ & 2.380 & 4.420 & 3.400 & \\
\hline 1:A_6:H2 & 1:A_15:H1' & 2.125 & 3.946 & 3.035 & \\
\hline 1:A_6:H8 & 1:G_5:H1' & 2.301 & 4.274 & 3.288 & \\
\hline 1:A_6:H8 & 1:A_6:H1' & 3.059 & 5.682 & 4.371 & $200 \mathrm{~ms}$ \\
\hline 1:A_6:H8 & 1:A_6:H2' & 3.633 & 6.000 & 5.190 & $200 \mathrm{~ms}$ \\
\hline 1:A_6:H8 & 1:A_6:H3' & 1.971 & 3.660 & 2.815 & \\
\hline $1: G \_7: H 8$ & 1:A_6:H2' & 2.883 & 5.354 & 4.118 & \\
\hline 1:G_7:H8 & 1:A_6:H3' & 1.561 & 2.899 & 2.230 & \\
\hline 1:G_7:H8 & $1: G \_7: H 1^{\prime}$ & 2.463 & 4.575 & 3.519 & \\
\hline $1: G_{-} 7: H 2^{\prime}$ & 1:G_8:H8 & 1.659 & 3.081 & 2.370 & \\
\hline 1:G_7:H3' & $1: G \_7: H 8$ & 1.739 & 5.000 & 2.484 & \\
\hline $1: \mathrm{G}_{-} 7: \mathrm{H} 3^{\prime}$ & 1:G_8:H8 & 1.711 & 5.000 & 2.444 & $*$ \\
\hline 1:G_8:H8 & $1: G \_7: H 1 '$ & 2.743 & 5.095 & 3.919 & \\
\hline $1: G \_8: H 8$ & 1:G_8:H1' & 2.716 & 5.043 & 3.879 & \\
\hline 1:G_8:H1' & $1: G \_8: H 4 '$ & 2.230 & 4.142 & 3.186 & \\
\hline 1:G_8:H1' & 1:C_9:H6 & 2.701 & 5.017 & 3.859 & $200 \mathrm{~ms}$ \\
\hline 1:G_8:H2' & $1: G \_8: H 8$ & 3.177 & 5.900 & 4.539 & \\
\hline 1:G_8:H2' & 1:G_8:H1' & 1.807 & 3.356 & 2.582 & \\
\hline 1:G_8:H3' & 1:G_8:H8 & 1.805 & 3.352 & 2.578 & \\
\hline 1:G_8:H3' & 1:C_9:H6 & 2.366 & 4.393 & 3.380 & \\
\hline 1:C_9:H6 & $1: G \_8: H 2 '$ & 1.617 & 3.002 & 2.309 & \\
\hline 1:C_9:H6 & 1:C_9:H5 & 1.691 & 3.140 & 2.415 & \\
\hline 1:C_9:H1' & $1: G \_8: H 2 '$ & 2.464 & 4.577 & 3.521 & \\
\hline 1:C_9:H1' & 1:C_9:H6 & 2.432 & 4.517 & 3.475 & \\
\hline 1:C_9:H1' & 1:C_9:H3' & 2.440 & 4.532 & 3.486 & \\
\hline 1:C_9:H1' & 1:U3'_10:H6 & 2.798 & 5.196 & 3.997 & \\
\hline 1:C_9:H2' & 1:C_9:H1' & 1.793 & 3.330 & 2.562 & \\
\hline 1:C_9:H3' & 1:C_9:H6 & 1.772 & 3.290 & 2.531 & \\
\hline 1:C_9:H3' & 1:U3'_10:H6 & 2.322 & 4.313 & 3.317 & \\
\hline 1:C_9:H4' & 1:C_9:H1' & 2.346 & 4.357 & 3.351 & \\
\hline 1:U3'_10:H5 & 1:C_9:H2' & 2.333 & 4.332 & 3.332 & \\
\hline 1:U3'_10:H6 & 1:C_9:H2' & 1.688 & 3.134 & 2.411 & \\
\hline 1:U3'_10:H6 & 1:U3'_10:H5 & 1.721 & 3.196 & 2.459 & \\
\hline 1:U3'_10:H1' & 1:U3'_10:H6 & 3.162 & 5.873 & 4.518 & \\
\hline 1:U3'_10:H1' & 1:U3'_10:H2' & 2.021 & 3.753 & 2.887 & \\
\hline 1:U3'_10:H2' & 1:U3'_10:H6 & 2.183 & 4.055 & 3.119 & \\
\hline 1:U3'_10:H3' & 1:U3'_10:H6 & 1.709 & 3.175 & 2.442 & \\
\hline 1:U3'_10:H3' & 1:U3'_10:H1' & 2.296 & 4.265 & 3.280 & \\
\hline 1:U3'_10:H5 & 1:C_9:H5 & 2.574 & 4.780 & 3.677 & $200 \mathrm{~ms}$ \\
\hline 1:U3'_10:H5 & 1:C_9:H6 & 3.162 & 5.872 & 4.517 & $200 \mathrm{~ms}$ \\
\hline 1:U3'_10:H5 & 1:C_9:H3' & 3.135 & 5.823 & 4.479 & $200 \mathrm{~ms}$ \\
\hline 1:U3'_10:H1' & 1:C_9:H2' & 2.729 & 5.069 & 3.899 & $200 \mathrm{~ms}$ \\
\hline 1:G5'_11:H8 & 1:G5'_11:H1' & 2.454 & 4.558 & 3.506 & \\
\hline 1:G5'_11:H8 & 1:G5'_11:H2' & 2.705 & 5.024 & 3.865 & \\
\hline
\end{tabular}




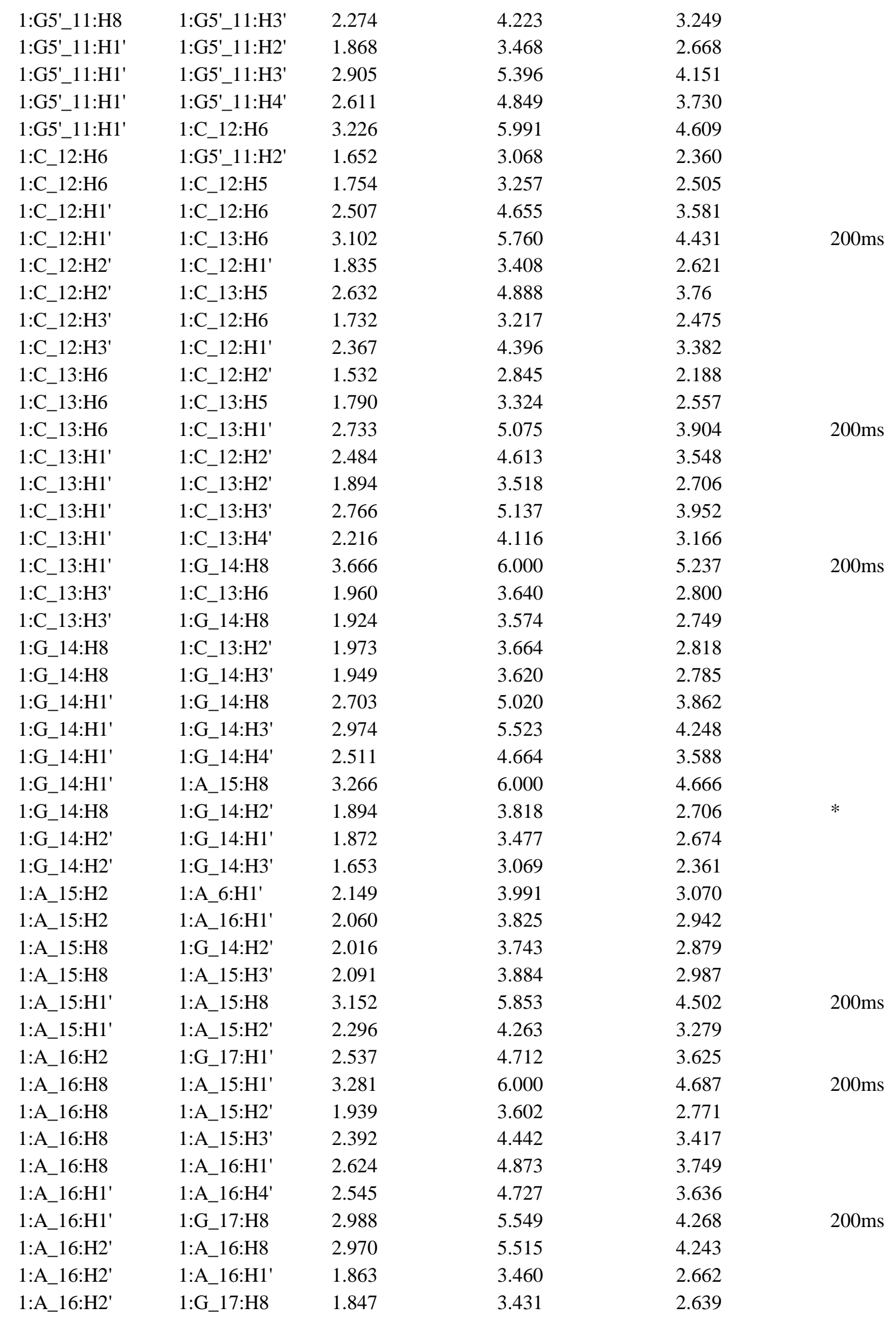




\begin{tabular}{|c|c|c|c|c|c|}
\hline 1:A_16:H3' & 1:A_16:H8 & 2.286 & 4.245 & 3.265 & \\
\hline 1:A_16:H3' & 1:A_16:H2' & 1.717 & 3.188 & 2.453 & \\
\hline 1:A_16:H3' & 1:G_17:H8 & 1.661 & 3.085 & 2.373 & \\
\hline 1:G_17:H2' & 1:G_17:H8 & 2.034 & 5.000 & 2.906 & \\
\hline 1:G_17:H2' & 1:C_18:H6 & 1.602 & 2.975 & 2.288 & \\
\hline 1:G_17:H2' & 1:C_18:H1' & 2.748 & 5.103 & 3.925 & \\
\hline 1:G_17:H3' & 1:C_18:H6 & 1.820 & 3.379 & 2.599 & \\
\hline 1:C_18:H5 & 1:C_18:H6 & 1.697 & 3.151 & 2.424 & \\
\hline 1:C_18:H1' & 1:C_18:H3' & 2.338 & 4.343 & 3.341 & \\
\hline 1:C_18:H1' & 1:C_19:H6 & 2.892 & 5.371 & 4.132 & \\
\hline 1:C_18:H2' & 1:C_18:H6 & 2.570 & 4.772 & 3.671 & \\
\hline 1:C_18:H2' & 1:C_18:H1' & 1.889 & 3.509 & 2.699 & \\
\hline 1:C_18:H2' & 1:C_19:H6 & 1.686 & 3.132 & 2.409 & \\
\hline 1:C_18:H3' & 1:C_18:H6 & 1.672 & 3.105 & 2.389 & \\
\hline 1:C_18:H3' & 1:C_19:H6 & 1.868 & 5.000 & 2.668 & \\
\hline 1:C_18:H4' & 1:C_18:H1' & 2.141 & 3.976 & 3.058 & \\
\hline 1:C_19:H6 & 1:C_19:H5 & 1.720 & 3.195 & 2.457 & \\
\hline 1:C_19:H1' & 1:C_18:H2' & 2.798 & 5.196 & 3.997 & \\
\hline 1:C_19:H1' & 1:C_19:H6 & 2.728 & 5.067 & 3.897 & \\
\hline 1:C_19:H1' & 1:C_19:H3' & 2.404 & 4.465 & 3.434 & \\
\hline 1:C_19:H1' & 1:P3'_20:H8 & 3.145 & 5.841 & 4.493 & $200 \mathrm{~ms}$ \\
\hline 1:C_19:H2' & 1:C_19:H1' & 1.903 & 3.534 & 2.718 & \\
\hline 1:C_19:H3' & 1:C_19:H6 & 1.669 & 3.099 & 2.384 & \\
\hline 1:C_19:H4' & 1:C_19:H1' & 2.023 & 3.758 & 2.890 & \\
\hline 1:P3'_20:H8 & 1:C_19:H3' & 2.162 & 4.014 & 3.088 & \\
\hline 1:P3'_20:H8 & 1:P3'_20:H1' & 2.936 & 5.453 & 4.195 & \\
\hline 1:P3'_20:H1' & 1:P3'_20:H3' & 2.735 & 5.079 & 3.907 & \\
\hline 1:P3'_20:H1' & 1:P3'_20:H4' & 2.365 & 4.392 & 3.378 & \\
\hline 1:P3'_20:H2' & 1:P3'_20:H8 & 2.494 & 4.632 & 3.563 & \\
\hline 1:P3'_20:H2' & 1:P3'_20:H1' & 2.057 & 3.820 & 2.938 & \\
\hline 1:P3'_20:H3' & 1:P3'_20:H8 & 1.701 & 3.160 & 2.431 & \\
\hline 1:P3'_20:H2 & 1:G5'_1:H1' & 2.941 & 5.461 & 4.021 & $200 \mathrm{~ms}$ \\
\hline 1:C_19:H2' & 1:P3'_20:H8 & 1.902 & 3.533 & 2.717 & $200 \mathrm{~ms}$ \\
\hline 1:P3'_20:H8 & 1:C_19:H6 & 3.322 & 6.000 & 4.745 & $200 \mathrm{~ms}$ \\
\hline
\end{tabular}

Dihedral angle restraints

\begin{tabular}{|c|c|c|c|c|c|}
\hline \multirow[t]{2}{*}{ Atom 1} & \multirow[t]{2}{*}{ Atom 2} & Atom 3 & Atom 4 & \multirow[t]{2}{*}{ Lower limit } & \multirow[t]{2}{*}{ Upper limit } \\
\hline & & alpha $\quad 03^{\prime}-P-O$ & A-form & & \\
\hline 1:G5'_1:O3' & $1: G \_2: P$ & $1: G \_2: O 5 '$ & 1:G_2:C5' & -120 & 120 \\
\hline 1:G_2:O3' & 1:U_3:P & 1:U_3:O5' & 1:U_3:C5' & -120 & 120 \\
\hline 1:A_6:O3' & $1: G \_7: P$ & 1:G_7:O5' & 1:G_7:C5' & -120 & 120 \\
\hline 1:G_7:O3' & 1:G_8:P & $1: G_{-} 8: O 5 '$ & 1:G_8:C5' & -120 & 120 \\
\hline 1:G_8:O3' & 1:C_9:P & 1:C_9:O5' & 1:C_9:C5' & -120 & 120 \\
\hline 1:C_9:O3' & 1:U3'_10:P & 1:U3'_10:O5' & 1:U3'_10:C5' & -120 & 120 \\
\hline
\end{tabular}




\begin{tabular}{|c|c|c|c|c|c|}
\hline 1:G5'_11:O3' & 1:C_12:P & 1:C_12:O5' & 1:C_12:C5' & -120 & 120 \\
\hline 1:C_12:O3' & $1: C_{-} 13: P$ & 1:C_13:O5' & 1:C_13:C5' & -120 & 120 \\
\hline 1:A_16:O3' & $1: G \_17: P$ & 1:G_17:O5' & 1:G_17:C5' & -120 & 120 \\
\hline 1:G_17:O3' & $1: C_{-} 18: \mathrm{P}$ & 1:C_18:O5' & 1:C_18:C5' & -120 & 120 \\
\hline 1:C_18:O3' & $1: C_{-} 19: P$ & 1:C_19:O5' & 1:C_19:C5' & -120 & 120 \\
\hline \multirow[t]{2}{*}{ 1:C_19:O3' } & 1:P3'_20:P & 1:P3'_20:O5' & 1:P3'_20:C5' & -120 & 120 \\
\hline & \multicolumn{5}{|c|}{ beta $\quad$ P-O5'-C5'-C4'; A-form 178} \\
\hline 1:G_2:P & $1: G \_2: O 5 '$ & $1: G \_2: C 5{ }^{\prime}$ & 1:G_2:C4' & 150 & -150 \\
\hline 1:U_3:P & 1:U_3:O5' & 1:U_3:C5' & 1:U_3:C4' & 150 & -150 \\
\hline $1: G_{-} 7: P$ & $1: G_{-} 7: 05{ }^{\prime}$ & $1: G_{-} 7: C 5{ }^{\prime}$ & $1: G_{-} 7: C 4$ & 150 & -150 \\
\hline 1:G_8:P & $1: G \_8: O 5 '$ & $1: G \_8: C 5{ }^{\prime}$ & 1:G_8:C4' & 150 & -150 \\
\hline 1:C_9:P & 1:C_9:O5' & 1:C_9:C5' & 1:C_9:C4' & 150 & -150 \\
\hline 1:C_12:P & 1:C_12:O5' & $1: C_{-} 12: \mathrm{C}^{\prime}$ & 1:C_12:C4' & 150 & -150 \\
\hline $1: C \_13: P$ & 1:C_13:O5' & 1:C_13:C5' & 1:C_13:C4' & 150 & -150 \\
\hline $1: G \_17: P$ & 1:G_17:O5' & 1:G_17:C5' & 1:G_17:C4' & 150 & -150 \\
\hline 1:C_18:P & 1:C_18:O5' & 1:C_18:C5' & 1:C_18:C4' & 150 & -150 \\
\hline \multirow[t]{2}{*}{ 1:C_19:P } & 1:C_19:O5' & 1:C_19:C5' & 1:C_19:C4' & 150 & -150 \\
\hline & \multicolumn{5}{|c|}{ gamma O5'-C5'-C4'-C3'; A-form 54} \\
\hline 1:G5'_1:O5' & 1:G5'_1:C5' & 1:G5'_1:C4' & 1:G5'_1:C3' & 30 & 90 \\
\hline 1:G_2:O5' & $1: G \_2: C 5^{\prime}$ & $1: G \_2: C 4$ & 1:G_2:C3' & 30 & 90 \\
\hline 1:U_3:O5' & 1:U_3:C5' & 1:U_3:C4' & 1:U_3:C3' & 30 & 90 \\
\hline $1: G \_7: O 5 '$ & $1: G_{-} 7: C 5^{\prime}$ & 1:G_7:C4' & 1:G_7:C3' & 30 & 90 \\
\hline 1:G_8:O5' & 1:G_8:C5' & 1:G_8:C4' & 1:G_8:C3' & 30 & 90 \\
\hline 1:C_9:O5' & 1:C_9:C5' & 1:C_9:C4' & 1:C_9:C3' & 30 & 90 \\
\hline 1:G5'_11:O5' & 1:G5'_11:C5' & 1:G5'_11:C4' & 1:G5'_11:C3' & 30 & 90 \\
\hline 1:C_12:O5' & 1:C_12:C5' & 1:C_12:C4' & 1:C_12:C3' & 30 & 90 \\
\hline 1:C_13:O5' & 1:C_13:C5' & 1:C_13:C4' & 1:C_13:C3' & 30 & 90 \\
\hline 1:G_17:O5' & 1:G_17:C5' & 1:G_17:C4' & 1:G_17:C3' & 30 & 90 \\
\hline 1:C_18:O5' & 1:C_18:C5' & 1:C_18:C4' & 1:C_18:C3' & 30 & 90 \\
\hline \multirow[t]{2}{*}{ 1:C_19:O5' } & 1:C_19:C5' & 1:C_19:C4' & 1:C_19:C3' & 30 & 90 \\
\hline & \multicolumn{5}{|c|}{ delta C5'-C4'-C3'-O3'; A-form 82} \\
\hline 1:G5'_1:C5' & 1:G5'_1:C4' & 1:G5'_1:C3' & 1:G5'_1:O3' & 55 & 115 \\
\hline 1:G_2:C5' & $1: G \_2: C 4 '$ & 1:G_2:C3' & 1:G_2:O3' & 55 & 115 \\
\hline 1:U_3:C5' & 1:U_3:C4' & 1:U_3:C3' & 1:U_3:O3' & 55 & 115 \\
\hline $1: G \_4: C 5^{\prime}$ & $1: G \_4: C 4 '$ & $1: G_{-} 4: C 3 '$ & 1:G_4:O3' & 55 & 115 \\
\hline 1:G_5:C5' & $1: G \_5: C 4 '$ & 1:G_5:C3' & 1:G_5:O3' & 130 & -170 \\
\hline 1:A_6:C5' & 1:A_6:C4' & 1:A_6:C3' & 1:A_6:O3' & 55 & -170 \\
\hline $1: G_{-} 7: C 5^{\prime}$ & $1: G_{-} 7: C 4$ & 1:G_7:C3' & 1:G_7:O3' & 55 & 115 \\
\hline 1:G_8:C5' & 1:G_8:C4' & 1:G_8:C3' & 1:G_8:O3' & 55 & 115 \\
\hline 1:C_9:C5' & 1:C_9:C4' & 1:C_9:C3' & 1:C_9:O3' & 55 & 115 \\
\hline 1:U3'_10:C5' & 1:U3'_10:C4' & 1:U3'_10:C3' & 1:U3'_10:O3' & 55 & -170 \\
\hline 1:G5'_11:C5' & 1:G5'_11:C4' & 1:G5'_11:C3' & 1:G5'_11:O3' & 55 & 115 \\
\hline 1:C_12:C5' & 1:C_12:C4' & 1:C_12:C3' & 1:C_12:O3' & 55 & 115 \\
\hline 1:C_13:C5' & 1:C_13:C4' & 1:C_13:C3' & 1:C_13:O3' & 55 & 115 \\
\hline 1:G_14:C5' & 1:G_14:C4' & 1:G_14:C3' & 1:G_14:O3' & 55 & -170 \\
\hline 1:A_15:C5' & 1:A_15:C4' & 1:A_15:C3' & 1:A_15:O3' & 55 & 115 \\
\hline 1:A_16:C5' & 1:A_16:C4' & 1:A_16:C3' & 1:A_16:O3' & 55 & 115 \\
\hline 1:G_17:C5' & 1:G_17:C4' & 1:G_17:C3' & 1:G_17:O3' & 55 & 115 \\
\hline
\end{tabular}




\begin{tabular}{|c|c|c|c|c|c|}
\hline 1:C_18:C5' & 1:C_18:C4' & 1:C_18:C3' & 1:C_18:O3' & 55 & 115 \\
\hline 1:C_19:C5' & 1:C_19:C4' & 1:C_19:C3' & 1:C_19:O3' & 55 & 115 \\
\hline 1:P3'_20:C5' & 1:P3'_20:C4' & 1:P3'_20:C3' & 1:P3'_20:O3' & 55 & -170 \\
\hline \multicolumn{6}{|c|}{ epsilon $\mathrm{C}^{\prime}$ '-C3'-O3'-P; A-form -153} \\
\hline 1:G5'_1:C4' & 1:G5'_1:C3' & 1:G5'_1:O3' & $1: G \_2: P$ & -180 & -100 \\
\hline $1: G \_2: C 4 '$ & 1:G_2:C3' & 1:G_2:O3' & $1: \mathrm{U} \_3: P$ & -180 & -100 \\
\hline $1: U_{-} 3: C 4 '$ & 1:U_3:C3' & 1:U_3:O3' & $1: G \_4: P$ & -180 & -100 \\
\hline $1: G \_7: C 4 '$ & 1:G_7:C3' & 1:G_7:O3' & $1: G \_8: P$ & -180 & -100 \\
\hline 1:G_8:C4' & 1:G_8:C3' & 1:G_8:O3' & 1:C_9:P & -180 & -100 \\
\hline 1:C_9:C4' & 1:C_9:C3' & 1:C_9:O3' & 1:U3'_10:P & -180 & -100 \\
\hline 1:G5'_11:C4' & 1:G5'_11:C3' & 1:G5'_11:O3' & $1: C_{-} 12: P$ & -180 & -100 \\
\hline 1:C_12:C4' & 1:C_12:C3' & 1:C_12:O3' & $1: C \_13: P$ & -180 & -100 \\
\hline 1:C_13:C4' & 1:C_13:C3' & 1:C_13:O3' & $1: G \_14: P$ & -180 & -100 \\
\hline 1:G_17:C4' & 1:G_17:C3' & 1:G_17:O3' & $1: C \_18: P$ & -180 & -100 \\
\hline 1:C_18:C4' & 1:C_18:C3' & 1:C_18:O3' & 1:C_19:P & -180 & -100 \\
\hline 1:C_19:C4' & 1:C_19:C3' & 1:C_19:O3' & 1:P3'_20:P & -180 & -100 \\
\hline \multicolumn{6}{|c|}{ zeta C3'-O3'-P-O5'; A-form -71 } \\
\hline 1:G5'_1:C3' & 1:G5'_1:O3' & $1: G \_2: P$ & 1:G_2:O5' & -120 & 120 \\
\hline 1:G_2:C3' & 1:G_2:O3' & $1: U \_3: P$ & 1:U_3:O5' & -120 & 120 \\
\hline 1:U_3:C3' & 1:U_3:O3' & $1: G \_4: P$ & 1:G_4:O5' & -120 & 120 \\
\hline 1:G_7:C3' & $1: G_{-} 7: 03^{\prime}$ & 1:G_8:P & 1:G_8:O5' & -120 & 120 \\
\hline 1:G_8:C3' & 1:G_8:O3' & 1:C_9:P & 1:C_9:O5' & -120 & 120 \\
\hline 1:C_9:C3' & 1:C_9:O3' & 1:U3'_10:P & 1:U3'_10:O5' & -120 & 120 \\
\hline 1:G5'_11:C3' & 1:G5'_11:O3' & $1: C \_12: P$ & 1:C_12:O5' & -120 & 120 \\
\hline 1:C_12:C3' & 1:C_12:O3' & 1:C_13:P & 1:C_13:O5' & -120 & 120 \\
\hline 1:C_13:C3' & 1:C_13:O3' & $1: G \_14: P$ & 1:G_14:O5' & -120 & 120 \\
\hline 1:G_17:C3' & 1:G_17:O3' & $1: C \_18: P$ & 1:C_18:O5' & -120 & 120 \\
\hline 1:C_18:C3' & 1:C_18:O3' & $1: C \_19: P$ & 1:C_19:O5' & -120 & 120 \\
\hline 1:C_19:C3' & 1:C_19:O3' & 1:P3'_20:P & 1:P3'_20:O5' & -120 & 120 \\
\hline \multicolumn{6}{|c|}{ chi Glycosidic torsions; A-form -158 } \\
\hline 1:G5'_1:O4' & 1:G5'_1:C1' & 1:G5'_1:N9 & 1:G5'_1:C4 & 150 & -130 \\
\hline $1: G \_2: O 4 '$ & 1:G_2:C1' & 1:G_2:N9 & $1: G \_2: C 4$ & 150 & -130 \\
\hline 1:U_3:O4' & 1:U_3:C1' & $1: \mathrm{U} \_3: \mathrm{N} 1$ & 1:U_3:C2 & 150 & -130 \\
\hline 1:G_7:O4' & $1: G_{-} 7: C 1^{\prime}$ & $1: G \_7: N 9$ & $1: G \_7: C 4$ & 150 & -130 \\
\hline 1:G_8:O4' & 1:G_8:C1' & 1:G_8:N9 & 1:G_8:C4 & 150 & -130 \\
\hline 1:C_9:O4' & 1:C_9:C1' & 1:C_9:N1 & 1:C_9:C2 & 150 & -130 \\
\hline 1:U3'_10:O4' & 1:U3'_10:C1' & 1:U3'_10:N1 & 1:U3'_10:C2 & 150 & -130 \\
\hline 1:G5'_11:O4' & 1:G5'_11:C1' & 1:G5'_11:N9 & 1:G5'_11:C4 & 150 & -130 \\
\hline 1:C_12:O4' & 1:C_12:C1' & 1:C_12:N1 & $1: C_{-} 12: C 2$ & 150 & -130 \\
\hline 1:C_13:O4' & 1:C_13:C1' & 1:C_13:N1 & 1:C_13:C2 & 150 & -130 \\
\hline 1:G_17:O4' & 1:G_17:C1' & 1:G_17:N9 & $1: G \_17: C 4$ & 150 & -130 \\
\hline 1:C_18:O4' & 1:C_18:C1' & 1:C_18:N1 & 1:C_18:C2 & 150 & -130 \\
\hline 1:C_19:O4' & 1:C_19:C1' & 1:C_19:N1 & 1:C_19:C2 & 150 & -130 \\
\hline 1:P3'_20:O4' & 1:P3'_20:C1' & 1:P3'_20:N9 & 1:P3'_20:C4 & 150 & -130 \\
\hline
\end{tabular}


Table S2. Summary of NOE distance restraints.

\begin{tabular}{cccc}
\hline Nucleotide & Total & Intra-nucleotide & Inter-nucleotide \\
\hline G1 & 13 & 6 & 7 \\
G2 & 19 & 6 & 13 \\
U3 & 22 & 6 & 16 \\
G4 & 15 & 5 & 10 \\
G5 & 11 & 4 & 7 \\
A6 & 11 & 3 & 8 \\
G7 & 11 & 2 & 9 \\
G8 & 14 & 5 & 9 \\
C9 & 19 & 6 & 13 \\
U10 & 15 & 6 & 9 \\
G11 & 11 & 6 & 5 \\
C12 & 13 & 5 & 8 \\
C13 & 13 & 6 & 7 \\
G14 & 15 & 7 & 8 \\
A15 & 14 & 3 & 11 \\
A16 & 16 & 6 & 10 \\
G17 & 13 & 1 & 12 \\
C18 & 17 & 6 & 11 \\
C19 & 16 & 6 & 10 \\
P20 & 15 & 6 & 9 \\
\hline
\end{tabular}




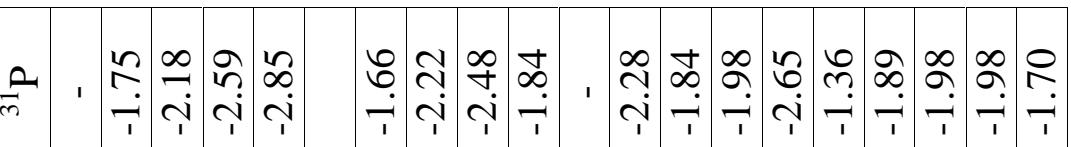

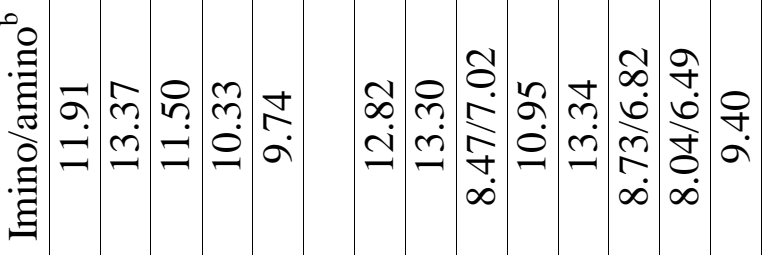

กิร

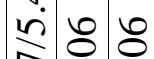

m.

ใุ

$\stackrel{\infty}{+} \infty \infty^{\infty}$

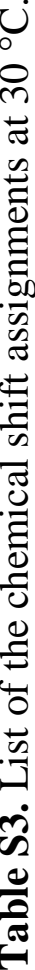

బิڤ

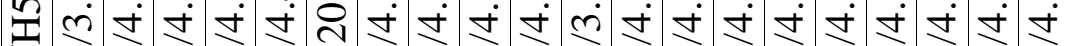

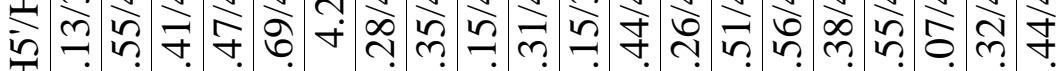

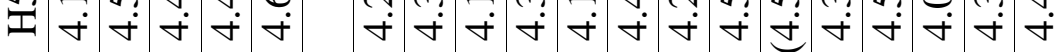

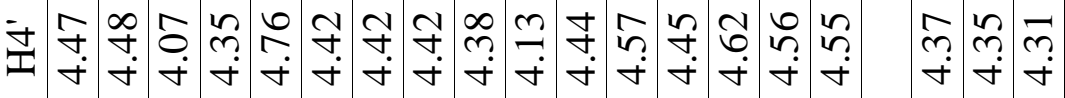

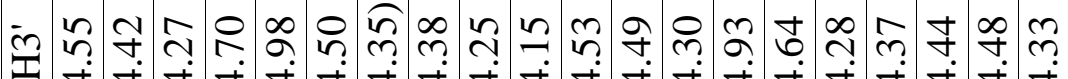

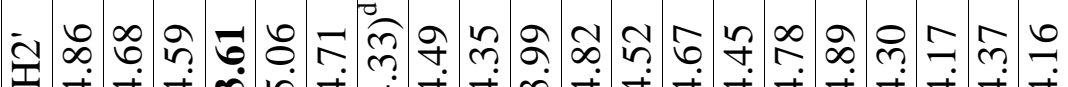

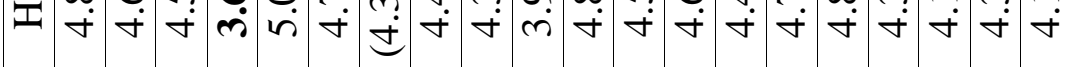

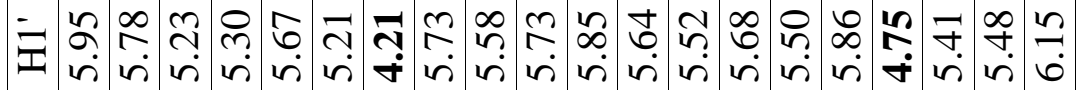

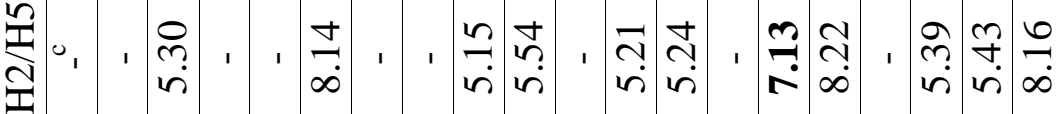

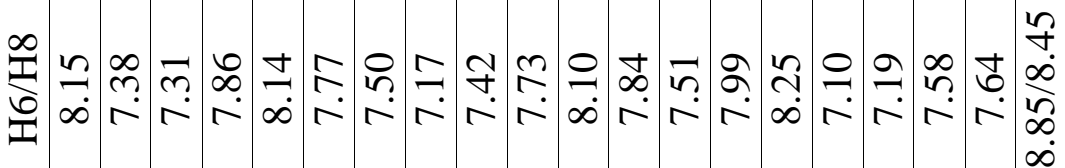

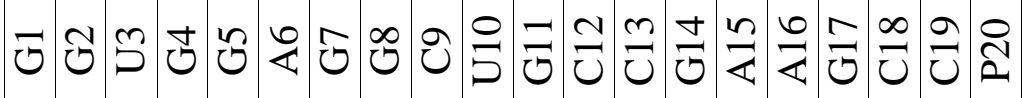

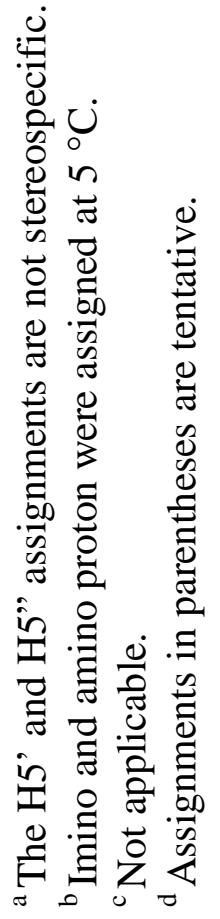




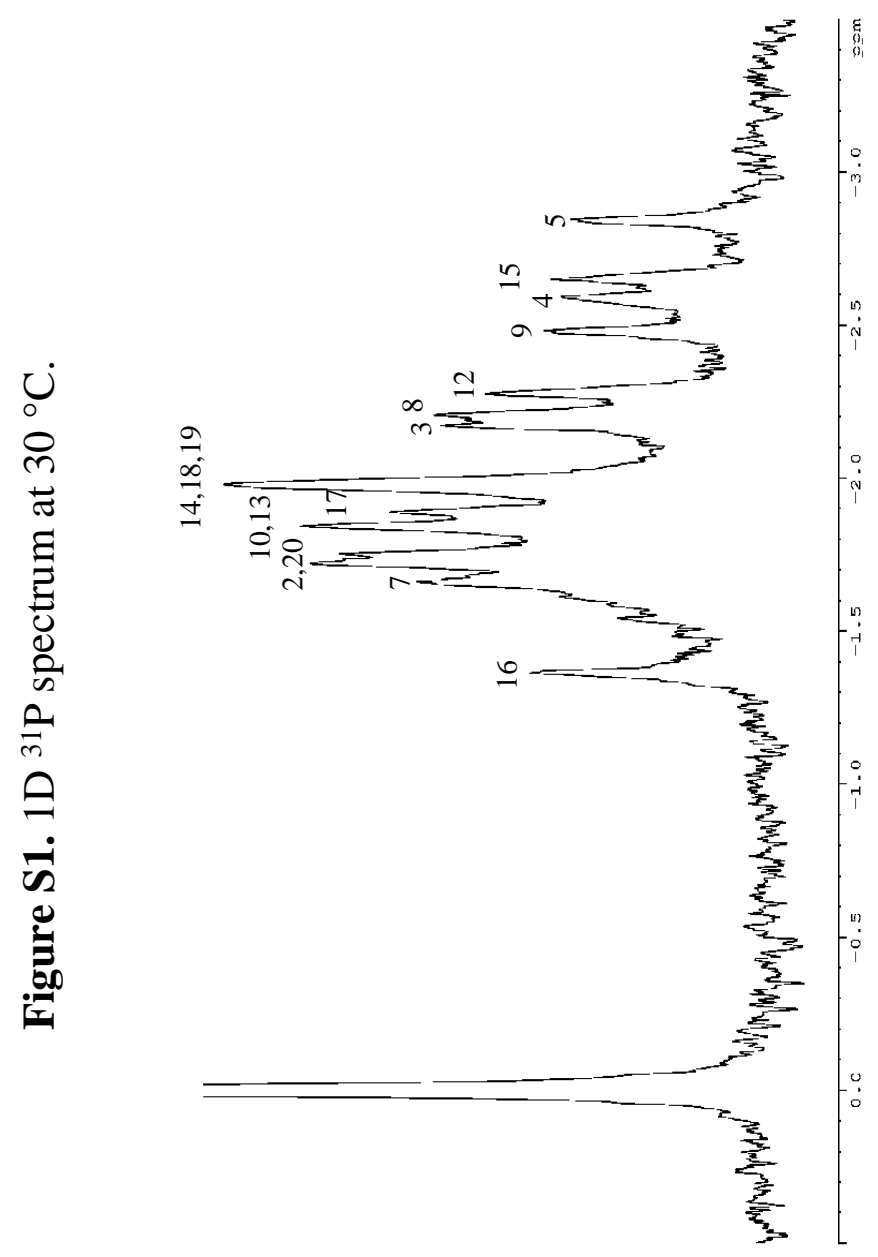




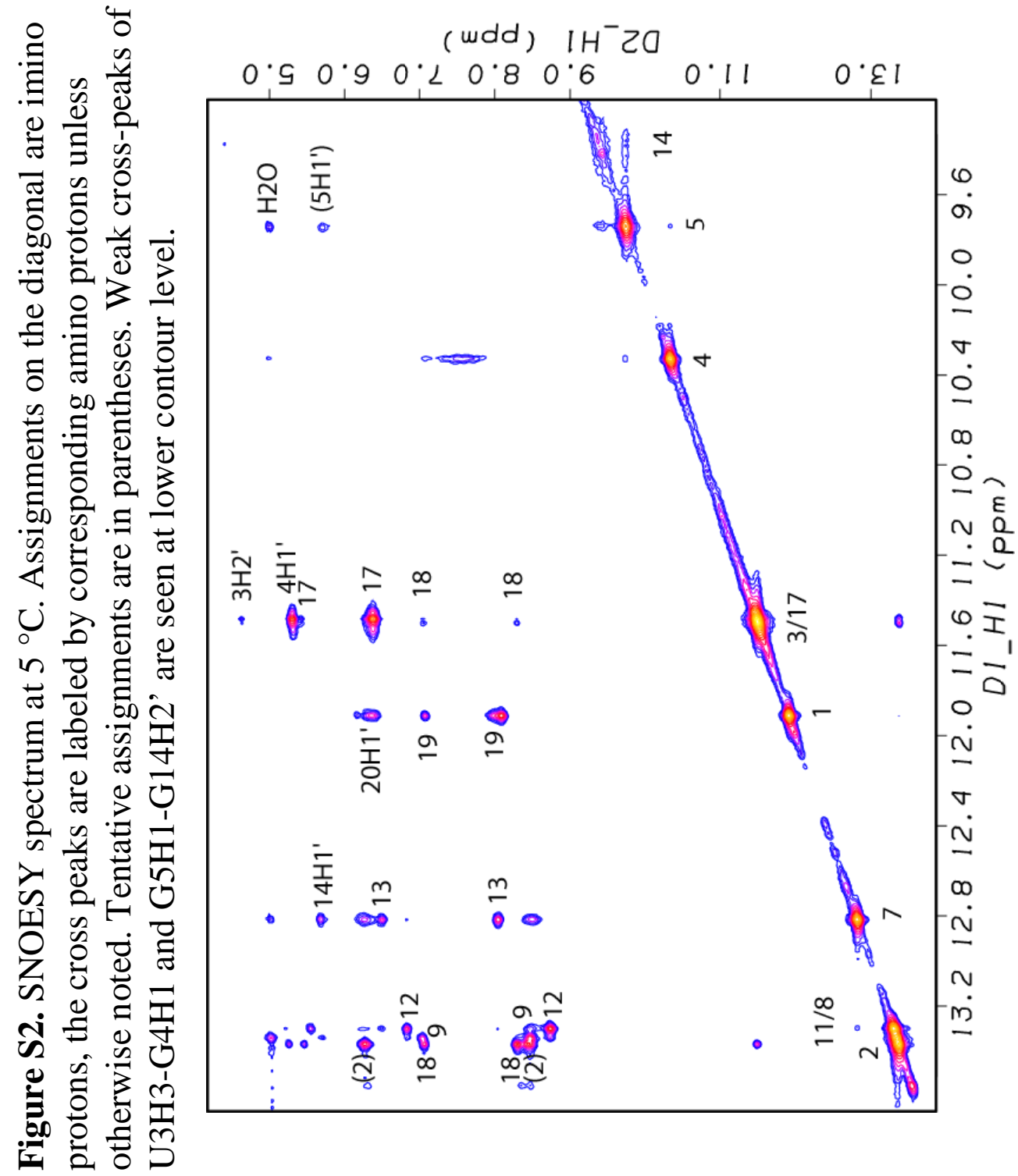




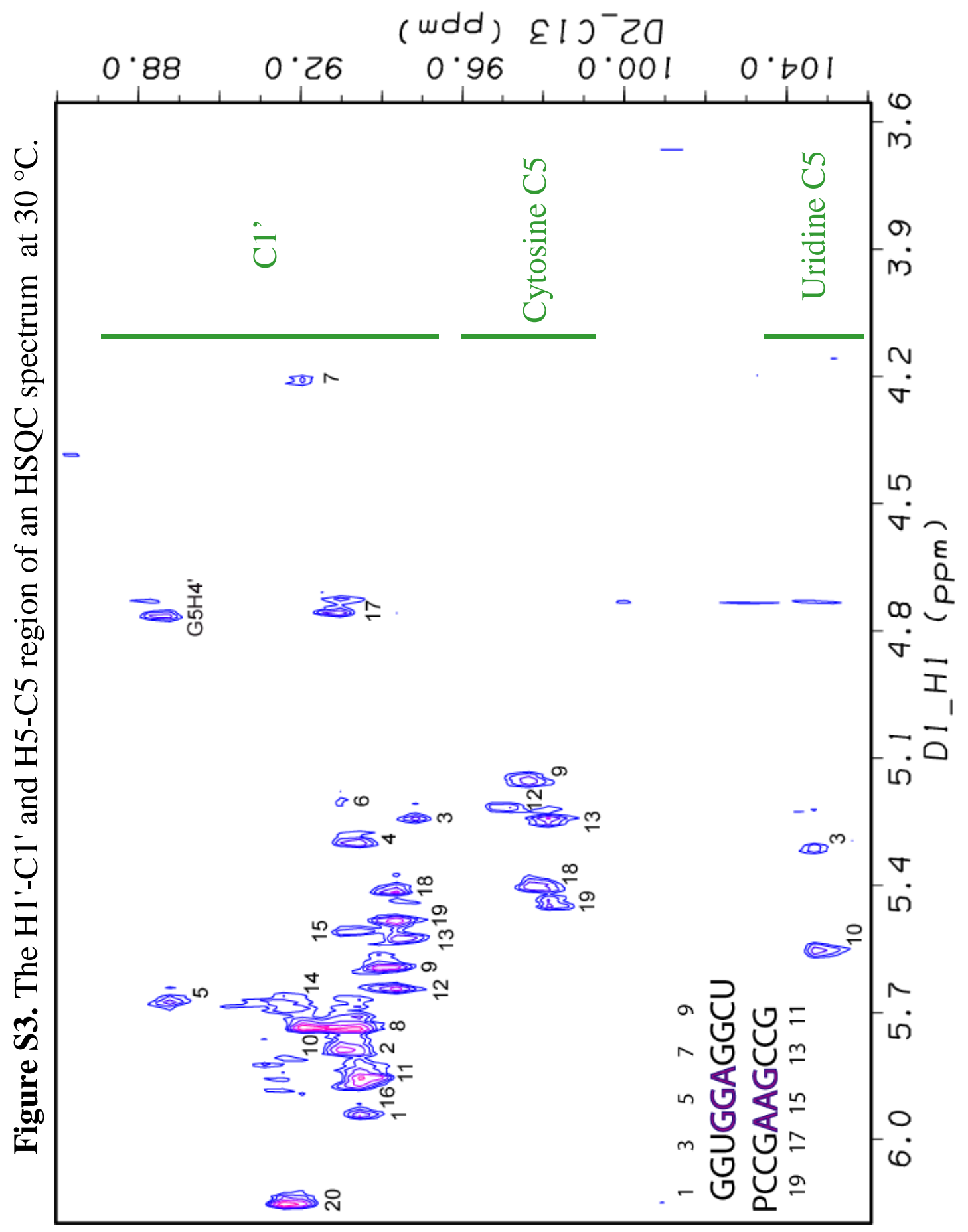




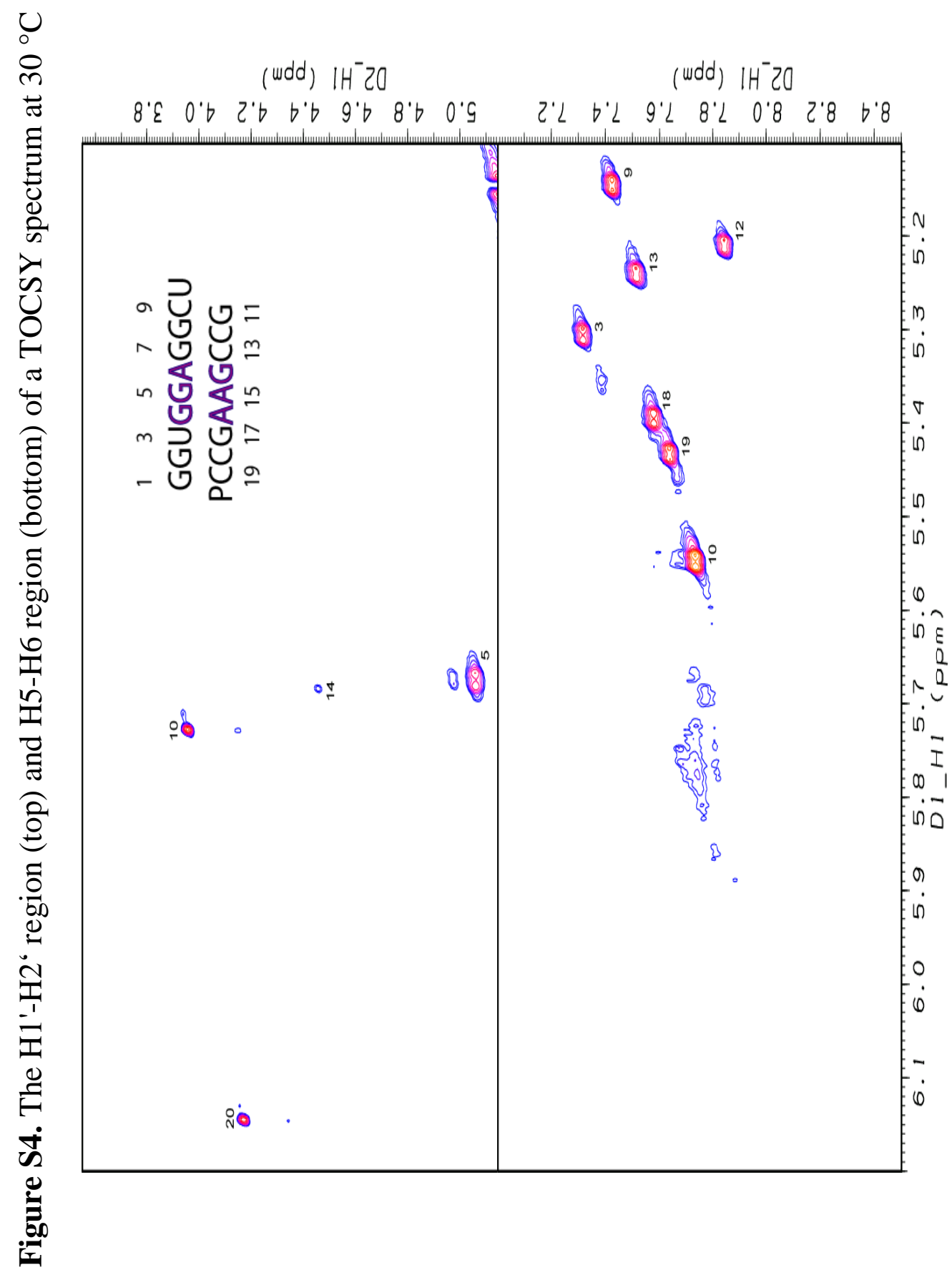




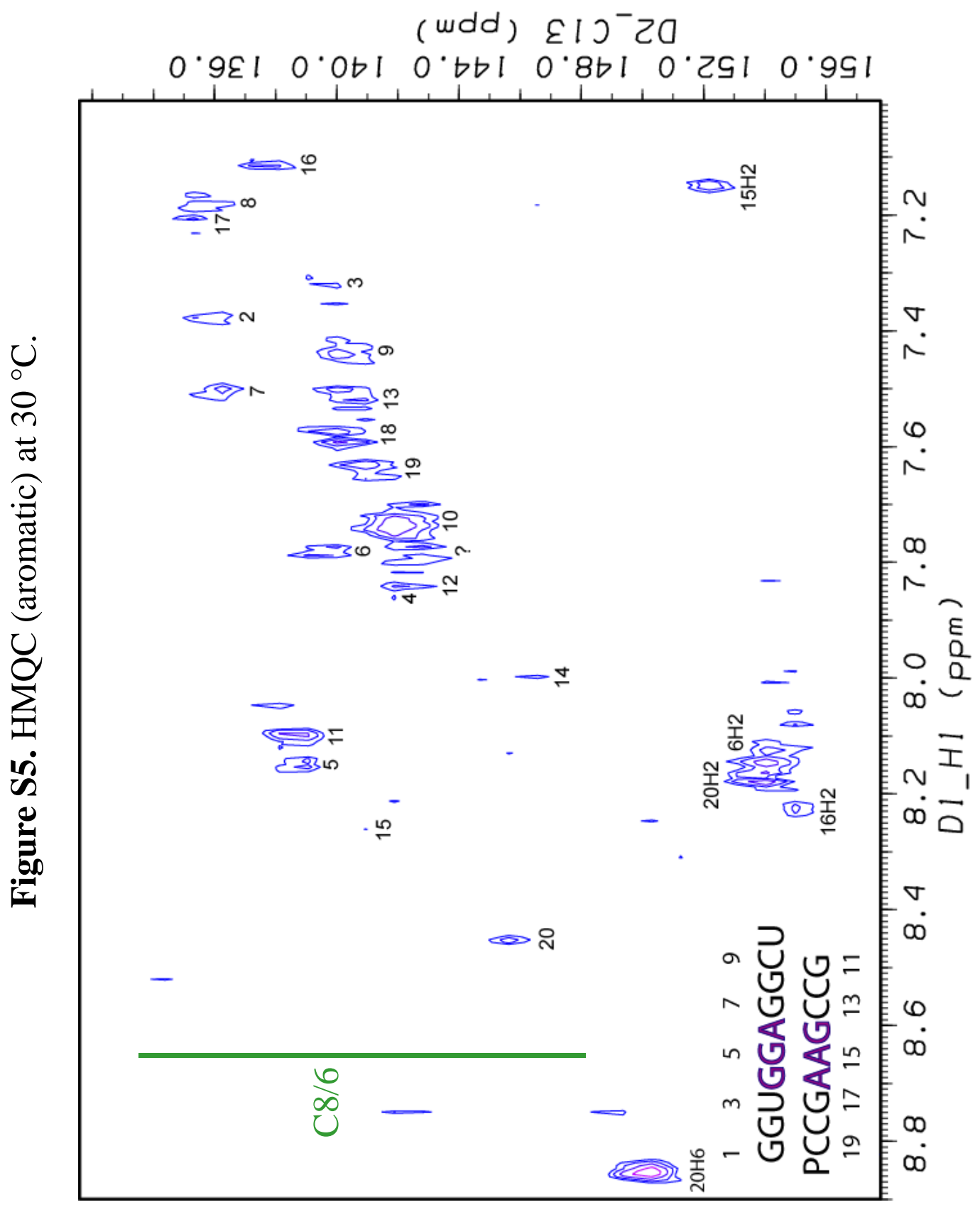




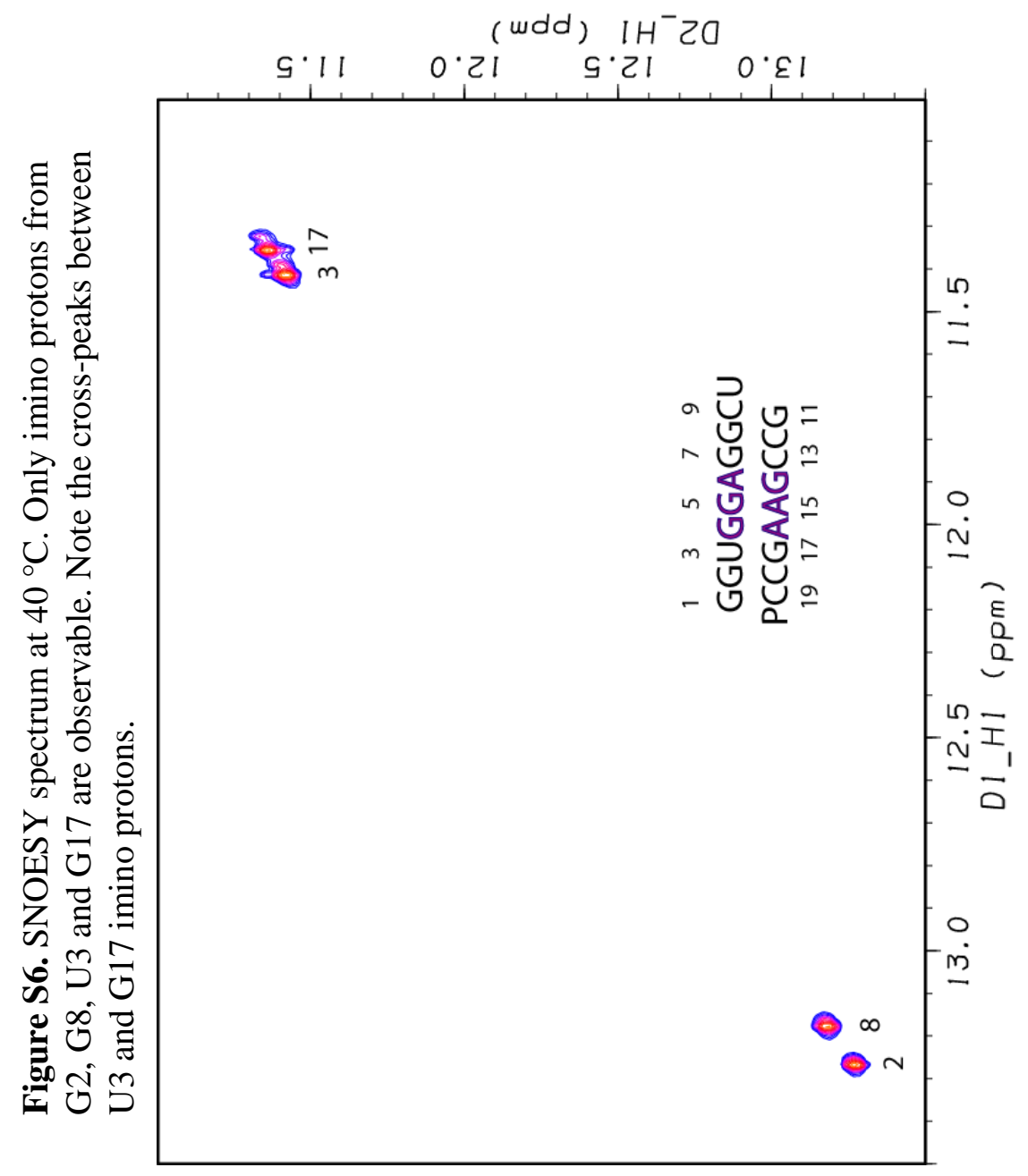

